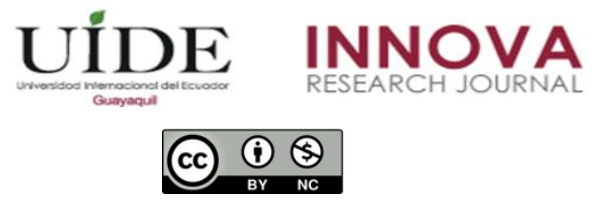

INNOVA Research Journal, ISSN 2477-9024

(Mayo-Agosto 2020). Vol. 5, No.2 pp. 140-156

DOI: https://doi.org/10.33890/innova.v5.n2.2020.1291

URL: http://revistas.uide.edu.ec/index.php/innova/index

Correo: innova@uide.edu.ec

\title{
La logística inversa como estrategia de diferenciación para los mercados dinámicos
}

\section{Reverse logistics as a differentiation strategy for dynamic markets}

Joselyn Estefanía Ruiz Sánchez

Mayiya Lisbeth González Illescas

http://orcid.org/0000-0002-5219-3807

Luis Pastor Carmenate Fuentes

Universidad Técnica de Machala, Ecuador

Autor por correspondencia: jeruiz_est@utmachala.edu.ec; mlgonzalez@utmachala.edu.ec; lcarmenate@utmachala.edu.ec

Fecha de recepción: 27 de diciembre de 2019 - Fecha de aceptación: 13 de abril de 2020

\section{Resumen}

Las exigencias de los mercados se ven reflejadas en estrictas regulaciones del cuidado ambiental, sumadas a la creciente concienciación de las personas y los gobiernos ha obligado a las organizaciones a involucrase como actores principales en el cuidado ambiental mediante la aplicación de la logística inversa. Este trabajo tiene por objetivo analizar la logística inversa como estrategia de diferenciación de las empresas exportadoras para competir en mercados dinámicos, a través de la revisión de trabajos de investigación de otros autores que han estudiado específicamente la implementación de la logística inversa en las empresas. Se aplica un enfoque cualitativo mediante los métodos teóricos: histórico-lógico, analítico-sintético e inductivodeductivo. Entre los resultados, se destaca que las empresas que han aplicado la logística inversa han mejorado su competitividad al reducir costos, reflejando credibilidad en el mercado por sus esfuerzos en minimizar la huella ambiental. Se concluye que la aplicación de las prácticas que abarca la logística inversa, aumenta las posibilidades de diferenciación de la competencia debido a que generan confianza y seguridad a los consumidores.

Palabras claves: estrategia de diferenciación; logística inversa; empresas exportadoras; mercados dinámicos; medio ambiente

\begin{abstract}
The requirements of the markets are reflected in strict regulations of environmental care, added to the growing awareness of individuals and governments, has forced organizations to become major players in environmental care through the application of reverse logistics. This paper aims to analyze reverse logistics as a strategy to differentiate exporting companies to compete in dynamic markets through the review of research papers by other authors who have specifically studied the implementation of reverse logistics in companies. A qualitative approach is applied using theoretical methods: historical-logical, analytical-synthetic and inductive-deductive. Among the results it is noted that companies that have applied reverse logistics have improved their competitiveness by reducing costs, reflecting credibility in the market for their efforts to minimize
\end{abstract}


the environmental footprint. It is concluded that the application of the practices covered by reverse logistics increases the possibilities of differentiation from the competition because they generate confidence and safety to the consumers.

Keywords: differentiation strategy; reverse logistics; export companies; dynamic markets; environment

\section{Introducción}

Los mercados internacionales presentan continuos cambios, situación que dificulta la competitividad de las empresas en el tiempo. El dinamismo de los mercados se refleja en la evolución de la tecnología, nuevas tendencias en los gustos y preferencias de los consumidores, las transformaciones de las actividades comerciales, entre otras manifestaciones.

En este sentido, el dinamismo del entorno impulsa a las organizaciones a crear capacidades para desarrollar ventajas que las diferencien de los competidores. Las empresas exportadoras, con el objetivo de ingresar o mantenerse en los mercados, deben acoplarse a las exigencias de los consumidores, como a los requerimientos legales y ambientales del país con el que hace negocios.

Actualmente, la responsabilidad de la empresa no se basa solo en ofrecer calidad y precios asequibles, sino ofrecer un valor agregado a los productos en base a factores ambientales. La creciente preocupación por la excesiva contaminación del entorno, ha fomentado el compromiso de las empresas a desarrollar la función de logística inversa, la cual de forma concreta implica la gestión de los desechos y productos devueltos para recuperar el mayor valor económico posible y reducir los residuos generados. De esta manera, la logística inversa se considera como una estrategia empresarial que favorece a la generación de ventajas competitivas sostenibles, permitiendo la reducción de costos y la contaminación ambiental, lo cual aporta a la credibilidad de las empresas y a la fidelización de sus clientes.

Esta investigación contribuye al análisis de la necesidad de las empresas exportadoras de responder de forma coherente a los nuevos escenarios, enfatizando en la importancia de adoptar la logística inversa como una estrategia innovadora, que aumenta las posibilidades de ganar prestigio vinculando el compromiso ambiental.

A tal efecto, se ha definido el propósito de analizar las prácticas de logística inversa como estrategia para diferenciarse de la competencia en mercados de continuo cambio. Para cumplir con el objetivo se utiliza un enfoque cualitativo mediante la revisión de quince artículos científicos que han abordado la implementación de la logística inversa en empresas pertenecientes a varios sectores industriales. Con el empleo de los métodos teóricos históricológico, analítico-sintético e inductivo-deductivo, se interpreta la caracterización de las prácticas organizacionales que definen la logística inversa y sus principales variables.

La estructura de este trabajo investigativo parte de una introducción seguida de la revisión de literatura que caracteriza a las estrategias de diferenciación, la logística inversa y sus variables operacionales, El apartado de resultados comprende el análisis de los principales hallazgos que señalan los trabajos seleccionados sobre la aplicación de logística inversa en 
empresas exportadoras. Por último, se abordan las conclusiones a manera de reflexiones sobre la relación entre logística inversa y la diferenciación que se proyecta al mercado, seguidas de las limitaciones y el planteamiento de futuras líneas de investigación.

\section{Desarrollo}

\section{Estrategia de diferenciación}

En un mercado cada vez más competitivo las empresas buscan la manera de distinguirse, así se intensifica la necesidad de aplicar estrategias de diferenciación sostenibles en los productos y servicios que sean perceptibles por los clientes.

Según Brüning, Masi, Jacobsen y Godsell (2018), la estrategia de diferenciación implica dotar al producto o servicio de elementos adicionales que apunten a la mejora continua, y que a la vez satisfagan las expectativas de los clientes, motivándolos a pagar un mayor precio.

La estrategia de diferenciación parte de la cadena de valor, donde se posibilita el robustecimiento de los factores asociados a la calidad y la innovación en los procesos y productos finales, con el fin de satisfacer plenamente las necesidades de los clientes y apuntar a una mejora continua.

Según Job y Nyongesa (2016), las estrategias de diferenciación comprenden la ingeniería de procesos y diseños innovadores, trabajando conjuntamente con la tecnología para obtener productos de calidad y beneficios superiores que la competencia.

Siguiendo los planteamientos de Semuel, Siagian y Octavia (2017), las empresas pueden alcanzar la diferenciación de la competencia a través de variables como el desempeño, la calidad del producto, la oferta de artículos, tiempo de entrega, los servicios (pre-venta y post-venta) y factores sociales y ambientales.

\section{La función logística en la empresa}

La evolución de las funciones de la logística parte de las actividades de transporte y el almacenamiento de productos (de la granja al mercado), inventarios y aspectos de la distribución física (Anca, 2019). Años más tarde Magee (1967) redefine a la logística como la integración de todos los procesos logísticos, se encarga del flujo de productos desde la obtención de la materia prima hasta cuando el artículo terminado llega al consumidor.

De acuerdo a Mejía, San Andrés y Paredes (2018), la gestión logística permite el flujo ágil de productos y servicios, elimina los excesos de stock, reduce costos, mejora los tiempos de entrega y genera confiabilidad en el bien ofertado.

Considerando la importancia de la función logística en las empresas exportadoras, frente a las constantes transformaciones de los mercados, de la revisión de literatura se extrae las definiciones contemporáneas de la logística para acercarnos al contexto de su operatividad. En en la tabla 1 se recogen los planteamientos de varios autores sobre logística. 


\section{Tabla 1}

Definiciones de Logística

\begin{tabular}{ll}
\hline Autores & Aportes sobre Logística \\
\hline Ballou (2004) & $\begin{array}{l}\text { Orientada hacia las necesidades del cliente. La logística crea valor para: los clientes, } \\
\text { proveedores, y coordina acciones. Su valor se expresa esencialmente en términos de tiempo } \\
\text { y lugar. }\end{array}$ \\
$\begin{array}{l}\text { Servera- } \\
\text { Francés (2010) }\end{array}$ & $\begin{array}{l}\text { Capacidades de integración entre todos los actores de suministro: proveedores, fabricantes, } \\
\text { distribuidores, operadoras logísticas, cliente, base de información, que agilizan las } \\
\text { actividades comerciales. }\end{array}$ \\
$\begin{array}{l}\text { Castellanos } \\
\text { (2015) }\end{array}$ & $\begin{array}{l}\text { Encargada de planificar y controlar de manera efectiva, el flujo y almacenamiento de los } \\
\text { productos o servicios desde su punto de origen hasta el punto de destino; para asegurar la } \\
\text { competitividad. }\end{array}$ \\
$\begin{array}{l}\text { Martínez, De } \\
\text { la Hoz, García } \\
\text { y Molina } \\
\text { (2017) }\end{array}$ & $\begin{array}{l}\text { Logística abarca el manejo del flujo de bienes y servicios, desde el aprovisionamiento de } \\
\text { matividades de compras, producción, distribución, almacenamiento y administración de } \\
\text { Philomena } \\
\text { (2018) }\end{array}$ \\
$\begin{array}{l}\text { inventarios. } \\
\text { Intervienen entradas y de salidas, manipulación de materiales, satisfacción de la demanda, } \\
\text { almacenamiento, diseño de redes y gestión de inventario. Conjuntamente con marketing, } \\
\text { operaciones, finanzas y tecnología de la información. }\end{array}$
\end{tabular}

Fuente: Elaboración propia apartir de los autores

Con lo expuesto, se deduce que la logística es un factor clave en cualquier organización, que permite desarrollar las actividades de manera planificada y coordinada durante el aprovisionamiento, producción y distribución. La función de la logística es asegurar a los clientes o consumidores la plena satisfacción de sus necesidades, cumpliendo con las condiciones de calidad total, costo asequible y en el tiempo adecuado.

La logística es un conjunto de factores que se involucran simultáneamente para obtener productos de calidad, buen servicio, reducir costos y cumplir en cuestiones de tiempo. Las principales áreas de la empresa son las que aseguran que el proceso logístico ese efectivo o deficiente. En el siguiente epígrafe se describen las áreas que cubre la logística.

\section{Áreas de acción de la logística}

La logística se encarga de las actividades de planificación, control y evaluación del desempeño de los procesos operativos de cada área de la organización. En cada área relacionada a la logística se identifican actividades específicas a desempeñar, sin embargo, con la partipicación de todas las partes se conforma una cadena colaborativa, que permite mejorar el rendimiento de las operaciones en general (Speranza, 2016). A continuación se analiza cada área de la logística en particular:

Aprovisionamiento, consiste en establecer los insumos requeridos, el precio, la calidad, la cantidad y la fecha en que se los necesita (González y Gutiérrez, 2018). Por medio del abastecimiento se efectua la planificación del volumen de producción (Brand, Monsalve y Rojas, 2017).

Esta obra se comparte bajo la licencia Creative Common Atribución-No Comercial 4.0 International (CC BY-NC 4.0) 
Producción, son las actividades programadas que contribuyen a la transformación de la materia prima en productos terminados, con el fin de atender las necesidades de los consumidores (Michlowicz y Smolińska, 2015).

Distribución, la distribución se sitúa en el flujo de elementos materiales, donde hace posible que la mercancía llegue desde el productor hasta el consumidor final (Sorlózano, 2018, pág. 181). Los movimientos de mercaderia se realizan dependiendo del producto, el tiempo de entrega y el transporte que se utilice.

En este sentido, Carro y Gónzalez (2013) mencionan las actividades que comprende cada área de la logistica. En la tabla 2 se recogen las tareas que desempeña cada área involucrada.

Tabla 2

Actividades de la logística dentro de la empresa

\begin{tabular}{lll}
\hline \multicolumn{1}{c}{ Aprovisionamiento } & \multicolumn{1}{c}{ Producción } & \multicolumn{1}{c}{ Distribución } \\
\hline Proceso de pedidos & Previsión de ventas & Transporte \\
Gestión de inventarios & Ingeniería y diseño & Tratamiento de mercancias \\
Transporte & Estrategia de producción & Equipos para la carga y descarga \\
Compras & Plan de materiales requeridos & Almacenaje y envase \\
Gestión de información & Maquinaria y equipo & Gestión de información \\
Tratamiento de mercancias & Gestión de inventarios de & Gestión de inventarios de bodega \\
& productos terminados &
\end{tabular}

Fuente: Elaboración propia apartir de Carro y Gónzalez (2013).

El aprovisionamiento empieza estableciendo los materiales o materia prima requerida para la producción, se selecciona a los proveedores y colaboradores con los que se va a trabajar, y se crea una base de información. Además, se crea un inventario para llevar un control de productos para evitar stocks innecesarios.

La producción es el proceso que comienza desde las solicitudes de pedidos en las cuales se establece el diseño y cantidades. Se implementa un plan de producción en el cual se especifica la maquinaria que se utilizará, los materiales, el tipo de envase y la mano de obra requerida; todos se complementan durante el desarrollo de la operación.

La distribución forma una cadena, cada eslabón interviene en la efectividad del proceso y para asegurar que el producto llegue en óptimas condiciones al cliente. La elección de los medios utilizados para la distribución es clave para satisfacer la demanda, tomando en cuenta las especificaciones (envases, embalaje) y reglamentos fijados en la normativa del país de destino.

\section{Logística inversa}

La ambición del hombre por obtener más ganancias y vivir cómodamente, ha provocado la degradación del medio ambiente. La implementación de nuevas tecnologías, los mercados 
consumistas y la competitividad entre empresas han traído consigo la idea de un mundo desechable (Santana, 2018).

Tras la preocupación por los altos niveles de contaminación ambiental los gobiernos, ONGs y consumidores, han creado consciencia ambiental e implementado rigurosas regulaciones para proteger al planeta y fomentar la economía circular. En el 2015 las Naciones Unidas estableció los Objetivos del Desarrollo Sostenible, metas que se plantea alcanzar hasta el 2030, las cuales abarca esferas económicas, sociales y ambientales, de aquí asociamos la importancia de la logística inversa, siendo parte fundamental para el desarrollo ambiental sostenible.

El Parlamento Europeo mediante una resolución adoptada en noviembre-2019, declaró a Europa en emergencia climática, lo que trae consigo la aplicación de regulaciones más estrictas para las industrias y demás medios contaminantes. Exigiendo a las empresas exportadoras que tienen relaciones comerciales con la UE a cumplir con medidas para el cuidado ambiental (DW, 2019).

En una sociedad cada vez más comprometida en temas sociales y ambientales, se requiere la participación de las empresas, mediante la incursión en procesos productivos sostenibles, responsabilidad corporativa y regulaciones ambientales, con el fin de contribuir para una economía circular (Bastos y Gallego, 2016).

Por otra parte, Palau (2015) señala que en el futuro la sostenibilidad logística se convertirá en un factor clave para la competitividad de las empresas, dado que los consumidores solicitarán la aplicación de energías más limpias y renovables en la producción. Además de cuestiones de responsabilidad social, que garantice la inclusión a la comunidad y beneficios económicos a sus empleados y colaboradores.

Las tendencias del desarrollo sostenible dan paso a que las empresas incursionen en el mejoramiento de sus procesos. La logística inversa realiza funciones específicas en la gestión de retornos, y proporciona un plus como estrategia de diferenciación de la organización en los mercados internacionales. Sirve para obtener competitividad e imagen corporativa, las empresas que participan en el cuidado del ambiente y desarrollo social son las preferidas en los mercados dinámicos (Maquera, 2012).

Para Saravanan, Sathiyagothai y Manigandan (2017), la logística inversa consiste en la dirección de retornos, una vez terminada la vida útil del bien, o cuando hay artículos defectuosos; se gestiona su regreso seguro a la empresa donde se los fabricó. Ya hecho el retorno de los artículos, se realiza una valoración de calidad, para la toma de decisiones sobre su nuevo destino, que pueden ser darle una nueva función o aplicar un procedimiento de eliminación con las medidas idóneas para proteger al ambiente (Lechner y Reimann, 2019).

Deacuerdo a Pagán, Tonelli, Silva y Da Silva (2017), la logística inversa comprende el diseño de envases reciclables para facilitar la recuperación y reutilización de los desechos, y así colaborar a la preservación del ambiente. 
Antonyová, Antony y Soewito (2016), identifican los factores estratégicos que tienen incidencia directa en la aplicación y desarrollo de la logística inversa: problemas ambientales, calidad, satisfacción del cliente, costos estratégicos y regulaciones legislativas de cada país. A continuación, en la figura 1 se presenta la secuencia gráfica de la logística inversa.

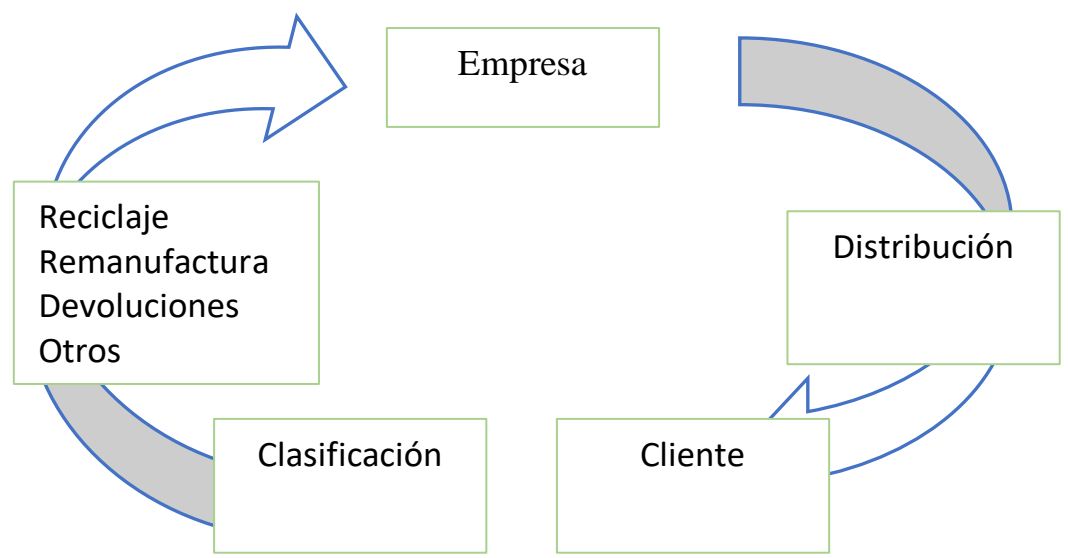

Figura 1. Circuito de la logística inversa

Se detalla el flujo de la logística inversa en el circuito cerrado post-venta que atraviesan los productos devueltos o los desechos de los mismos, al llegar nuevamente a la fábrica se aplica una clasificación para valorar las condiciones en que se encuentran los artículos que están reingresando para la toma de decisiones sobre la nueva función que cumplirán: reciclaje, reutilizar, devoluciones, remanufacturar, etc.

Las variables de la logística inversa le dan ventajas competitivas a la empresa, con un enfoque en reducción de costos y cuidado ambiental. A continuación, en la tabla 3 se recogen los criterios de tres autores al respecto de las variables operacionales que comprende la logística inversa.

Tabla 3

Variables de la logística inversa

\begin{tabular}{|c|c|c|}
\hline $\begin{array}{l}\text { Vega de la Cruz, Marrero y } \\
\text { Pérez (2017) }\end{array}$ & Huérfano y Meleán (2017) & Rubio y Jiménez-Parra (2014) \\
\hline Remanufactura & Recolección & Recolección \\
\hline Reciclaje & Devoluciones & Inspección y clasificación \\
\hline Reutilización & Transporte & Reusar \\
\hline $\begin{array}{l}\text { Gestión de residuos } \\
\text { (canibalización, vertedero) }\end{array}$ & Reciclaje & Remanufactura \\
\hline Devoluciones & Redistribución & Canibalización \\
\hline Análisis costo & Costos & - \\
\hline Transporte & - & - \\
\hline
\end{tabular}

Fuente: Elaboración propia a partir de los autores.

Posteriormente se define a cada variable operacional para analizar las actividades vinculadas a cada una y así mismo identificar su relevancia para la conservación del ambiente. 


\section{Tabla 4}

Variables de la logística inversa

\begin{tabular}{|c|c|c|c|}
\hline Factores & & Autores & Definiciones \\
\hline Reciclaje & & $\begin{array}{l}\text { Gullifa, Jatib, } \\
\text { Marcuzzi y Pérez } \\
\text { (2017) }\end{array}$ & $\begin{array}{l}\text { Permite utilizar los residuos de productos que ya cumplieron su } \\
\text { vida útil, dándoles a estos un nuevo uso y consiguiendo reducir } \\
\text { el uso de materias primas y energía. }\end{array}$ \\
\hline Devoluciones & & Jeszka (2014) & $\begin{array}{l}\text { Las devoluciones comprenden que el producto regrese al } \\
\text { proveedor, debido a que no cumple con las especificaciones, } \\
\text { están caducados o no funcionan. }\end{array}$ \\
\hline $\begin{array}{l}\text { Gestión } \\
\text { residuos }\end{array}$ & de & $\begin{array}{l}\text { Yousefloo y } \\
\text { Babazadeh } \\
\text { (2019) }\end{array}$ & $\begin{array}{l}\text { Se aplica en dependencia del tipo de desecho, los cuales pueden } \\
\text { ser: calcinación, digestión anaeróbica y vertedero, tomando las } \\
\text { consideraciones para proteger al ambiente. }\end{array}$ \\
\hline Remanufactura & & $\begin{array}{l}\text { Nurul, } \\
\text { Zwolinskia, } \\
\text { Mandil y } \\
\text { Brissaud (2017) }\end{array}$ & $\begin{array}{l}\text { Proceso que consiste en desmontar, reacondicionar, } \\
\text { reensamblar o reparar algún artículo con fallas, con el propósito } \\
\text { de crear uno nuevo a partir de sus desperdicios y así mantener } \\
\text { el valor del producto y reducir los desechos. }\end{array}$ \\
\hline $\begin{array}{l}\text { Análisis } \\
\text { costos }\end{array}$ & de & Alshubiri (2017) & $\begin{array}{l}\mathrm{La} \text { reducción de costos es significativa, mediante la } \\
\text { reutilización de materiales reciclados y la reventa de productos } \\
\text { a mercados menos exigentes. }\end{array}$ \\
\hline Reutilización & & $\begin{array}{l}\text { Singh, Shani, } \\
\text { Femal y Deif } \\
(2016)\end{array}$ & $\begin{array}{l}\text { Reutilizar los materiales usados y producir envases o embalajes } \\
\text { reutilizables, que aparte de ser la imagen del producto, deben } \\
\text { ser lo suficientemente resistentes para ser utilizados más de una } \\
\text { vez. }\end{array}$ \\
\hline $\begin{array}{l}\text { Redes } \\
\text { transporte }\end{array}$ & de & $\begin{array}{l}\text { Rubio, Jiménez- } \\
\text { Parra, Chamorro- } \\
\text { Mera y Miranda } \\
\text { (2019) }\end{array}$ & $\begin{array}{l}\text { Implica en establecer un diseño de redes que funcione de } \\
\text { manera sostenible para la recolección, tratamiento y traslado de } \\
\text { desechos hacia las empresas. }\end{array}$ \\
\hline
\end{tabular}

Fuente: Elaboración propia apartir de los autores.

Todos los factores anteriormente mencionados intervienen en el desarrollo efectivo de la logística inversa, teniendo como objetivo aplicar procesos conscientes para la conservación del entorno y reducir los costos operativos.

\section{Materiales y métodos}

Esta investigación tiene un enfoque cualitativo, de carácter descriptivo que consiste en analizar y caracterizar a la logística inversa que aplican las empresas. Se emplean los métodos teóricos histórico-lógico, analítico-sintético e inductivo-deductivo, para interpretar las prácticas organizacionales que definen la logística inversa y sus principales variables.

El estudio abarca la revisión de una selección de 15 artículos de investigación de otros autores que abordan a la logística inversa desde varios sectores industriales. Los artículos científicos seleccionados como fuente de información secundaria se obtuvieron de las bases de datos: Emerald, Taylor \& Francis, Elsevier, Scopus, Latindex, Scielo y Redalyc.

Los casos analizados corresponden a empresas exportadoras que han experimentado la función de logística inversa. Se enfatiza en las variables operacionales que han caracterizado sus 
prácticas organizacionales, y los resultados se enfocan en la relación de dichas variables con la generación de ventajas competitivas.

\section{Resultados y discusión}

Bajo la consideración del objetivo planteado y el marco teórico precedente, los resultados se presentan como la sistematización de los principales hallazgos expuestos en trabajos previos sobre la aplicación de la logística inversa. Tomando como premisa la caracterización de la estrategia de diferenciación, la logística inversa se analiza destacando las variables operacionales a partir de las cuales las empresas proyectan mejores resultados, en paralelo a la obtención de prestigio en el mercado por sus buenas prácticas ambientales

En la tabla 5, se recoge la sistematización de 15 artículos que estudiaron la situación de empresas exportadoras que emplearon la logística inversa:

\section{Tabla 5}

Logística inversa aplicada en empresas

\begin{tabular}{|c|c|c|c|}
\hline Autor/Año & Muestra/País & Variables & Resultados \\
\hline $\begin{array}{ll}\text { Bustos } & \text { F. } \\
(2015) & \end{array}$ & $\begin{array}{l}45 \text { industrias } \\
\text { manufactureras, } \\
\text { Venezuela }\end{array}$ & $\begin{array}{l}\text {-Remanufactura } \\
\text {-Reutilización } \\
\text {-Reparación } \\
\text {-Reciclaje } \\
\text {-Gestión de residuos }\end{array}$ & $\begin{array}{l}\text { Si bien las empresas en análisis } \\
\text { contemplan a la logística inversa como } \\
\text { una estrategia de diferenciación, se } \\
\text { destaca que existe poca atención en las } \\
\text { prácticas y operaciones de reciclaje, } \\
\text { canibalización, reutilización, control de } \\
\text { la contaminación y las certificaciones. }\end{array}$ \\
\hline Noé (2015) & $\begin{array}{l}6 \text { empresas, } \\
\text { Argentina }\end{array}$ & $\begin{array}{l}\text {-Reciclar } \\
\text {-Reutilizar } \\
\text {-Reducir }\end{array}$ & $\begin{array}{l}\text { Los autores del estudio identifican que el } \\
\text { flujo inverso está vinculado al sistema } \\
\text { 3R en el contexto de sustentabilidad y } \\
\text { responsabilidad social. Existe } \\
\text { cooperación con stakeholders para } \\
\text { aspectos de eficiencia, calidad e } \\
\text { innovación. Se destaca la relación } \\
\text { positiva entre la logística inversa y el } \\
\text { desempeño de las empresas. }\end{array}$ \\
\hline
\end{tabular}

\begin{tabular}{|c|c|c|c|}
\hline $\begin{array}{l}\text { Pagán, } \\
\text { Tonelli, } \\
\text { Silva y Da } \\
\text { Silva (2017) }\end{array}$ & $\begin{array}{l}3 \text { supermercados, } \\
\text { Sao Paulo-Brasil }\end{array}$ & $\begin{array}{l}\text {-Reciclaje } \\
\text {-Gestión de residuos } \\
\text { - Análisis costo }\end{array}$ & $\begin{array}{l}\text { Mediante el estudio de casos se } \\
\text { establece que la logística inversa reduce } \\
\text { los volúmenes de plástico y cartón en los } \\
\text { supermercados, se deja de producir al } \\
\text { ambiente materiales abióticos y bióticos, } \\
\text { y se evita la contaminación del agua y el } \\
\text { aire. Al mismo tiempo que realiza la } \\
\text { gestión de residuos, el reciclaje y } \\
\text { reventa, factores que mejoran la imagen } \\
\text { de la empresa, reducen costos y generan } \\
\text { ganancias adicionales. }\end{array}$ \\
\hline
\end{tabular}

\begin{tabular}{|c|c|c|c|}
\hline $\begin{array}{l}\text { Lopes, } \\
\text { D'Agosto, } \\
\text { Ferreira y }\end{array}$ & $\begin{array}{l}3 \text { grandes } \\
\text { almacenes, Brasil }\end{array}$ & $\begin{array}{l}\text {-Devoluciones } \\
\text {-Redes de transporte } \\
\text {-Análisis costo }\end{array}$ & $\begin{array}{l}\text { La logística inversa de las empresas en } \\
\text { estudio, reflejan su desempeño en } \\
\text { función de la gestión de entradas de } \\
\text { productos devueltos, el manejo de }\end{array}$ \\
\hline
\end{tabular}

Esta obra se comparte bajo la licencia Creative Common Atribución-No Comercial 4.0 International (CC BY-NC 4.0) 


\begin{tabular}{|c|c|c|}
\hline Autor/Año & Muestra/País & Resultados \\
\hline $\begin{array}{l}\text { Oliveira } \\
(2014)\end{array}$ & & $\begin{array}{l}\text { información sobre los motivos de los } \\
\text { retornos, la gestión de rendimiento } \\
\text { respecto a los costos de transporte y } \\
\text { almacenamiento; en consideración a la } \\
\text { ventaja competitiva que le aporta la } \\
\text { imagen corporativa. }\end{array}$ \\
\hline
\end{tabular}

\begin{tabular}{llll}
\hline Agrawal y & 208 organizaciones & -Remanufactura & Las empresas en análisis emplean \\
Singh (2019) & del sector & -Reutilización & prácticas de remanufactura, reutilización \\
& electrónico, India & -Reciclaje & y reciclaje. Impulsan su desarrollo \\
& & priorizando decisiones en el ámbito \\
& & operativo, mediante factores internos \\
& & como: costos, calidad y cantidades de \\
& & $\begin{array}{l}\text { productos retornados y externos como: } \\
\text { aspectos sociales, ambientales y } \\
\text { económicos. }\end{array}$
\end{tabular}

\begin{tabular}{llll}
\hline Brüning, & 7 estudios de casos, & -Análisis costos & El estudio señala las operaciones que se \\
Masi, & Dinamarca & -Reutilización & realizan en el ámbito de la logística \\
Jacobsen y & & -Remanufactura & inversa. Se centra en creación de valor, \\
Godsell & & & reducción de costos, aplicación de \\
(2018) & & habilidades técnicas de retorno, \\
& innovación, reutilización y garantía, \\
& para captar clientes.
\end{tabular}

\begin{tabular}{lll}
\hline Morgan, & 180 empresas del & -Análisis costo \\
Tokman, & sector industrial y & -Gestión de residuos \\
Richey y & logístico, Estados & \\
Defee (2018) & Unidos
\end{tabular}

De acuerdo a la experiencia de las empresas en análisis, se muestra a la logística inversa como una mediadora para conseguir la sostenibilidad ambiental. Se destaca como función que contribuye a proporcionar los recursos financieros, administrativos y físicos necesarios para llevar a cabo las actividades operativas inversas. Las conclusiones indican que se optimizaron los costos y se fortalecieron acciones de tipo ambiental.

\begin{tabular}{|c|c|c|c|}
\hline $\begin{array}{l}\text { Camargo, } \\
\text { Franco, } \\
\text { Chud y } \\
\text { Osorio } \\
(2017)\end{array}$ & $\begin{array}{l}\text { Sector } \\
\text { automovilístico de } \\
\text { llantas, Colombia }\end{array}$ & $\begin{array}{l}\text {-Reutilización } \\
\text {-Remanufactura } \\
\text {-Gestión de residuos }\end{array}$ & $\begin{array}{l}\text { El estudio se expresa en una red de } \\
\text { regreso al fabricante, consiste en } \\
\text { recolectar llantas para reencaucharlas, } \\
\text { co-procesarlas o triturarlas. Para } \\
\text { abastecer al mercado y gestionar los } \\
\text { desechos adecuadamente. Estos factores } \\
\text { generan ingresos extras y ayudan a la } \\
\text { reducción de emisiones de CO2. }\end{array}$ \\
\hline $\begin{array}{l}\text { Gullifa et al. } \\
\text { (2017) }\end{array}$ & $\begin{array}{l}\text { Industria cervecera, } \\
\text { Argentina }\end{array}$ & $\begin{array}{l}\text {-Reciclaje } \\
\text {-Reutilización } \\
\text {-Redes de transporte }\end{array}$ & $\begin{array}{l}\text { La logística inversa se plasma en el } \\
\text { reciclaje y reutilización de botellas para } \\
\text { abastecer de materiales reciclados a un } \\
\text { mercado de demanda creciente. Estas } \\
\text { empresas consiguen disminuir el } \\
\text { volumen de desechos sólidos, acortar } \\
\text { costos y gastos de transporte, crear }\end{array}$ \\
\hline
\end{tabular}




\begin{tabular}{|c|c|c|c|}
\hline Autor/Año & Muestra/País & Variables & Resultados \\
\hline & & & $\begin{array}{l}\text { ventajas competitivas y reducir la } \\
\text { contaminación ambiental. }\end{array}$ \\
\hline $\begin{array}{l}\text { Bottani, } \\
\text { Vignali, } \\
\text { Mosna y } \\
\text { Montanari } \\
\text { (2019) }\end{array}$ & $\begin{array}{l}\text { Minimercados, } \\
\text { supermercados e } \\
\text { hipermercados, } \\
\text { Emilia-Romagna, } \\
\text { Italia }\end{array}$ & $\begin{array}{l}\text {-Análisis de costo } \\
\text {-Redes de transporte } \\
\text {-Reciclaje }\end{array}$ & $\begin{array}{l}\text { Estas empresas se distinguen por } \\
\text { recolectar desperdicios de alimentos } \\
\text { envasados para reciclarlos y } \\
\text { almacenarlos, con el fin de que no } \\
\text { terminen en vertederos. El costo del } \\
\text { transporte de recolección es } \\
\text { considerable, pero el beneficio } \\
\text { ambiental y reducción de costos en la } \\
\text { remanufactura hace que lo compense } \\
\text { con una mejor reputación que se } \\
\text { visibiliza en el aumento en ventas. }\end{array}$ \\
\hline
\end{tabular}

\begin{tabular}{llll}
\hline Trochu, & Industria de la & -Reciclaje \\
Chaabane y & construcción, & & -Redes de transporte \\
Ouhimmu & Quebec-Canadá & & -Gestión de residuos
\end{tabular}

(2019)

El estudio demuestra que en Quebec existen regulaciones específicas sobre los desechos de construcción, mediante centros de recolección que valoran a la madera reciclada, con el fin de reducir los materiales de los vertederos, disminuir las emisiones de $\mathrm{CO} 2$ y lograr ecoeficiencia bajo incertidumbre.

\begin{tabular}{|c|c|c|c|}
\hline $\begin{array}{l}\text { Guarnieri, } \\
\text { Cerqueira- } \\
\text { Streit y } \\
\text { Batista } \\
(2020)\end{array}$ & $\begin{array}{llr}\text { Sector de la } & \text { de } \\
\text { industria } & & \text { del } \\
\text { embalaje, } & \text { Brasil }\end{array}$ & $\begin{array}{l}\text {-Reutilización de } \\
\text { envases o embalaje }\end{array}$ & $\begin{array}{l}\text { La logística inversa se articula bajo la } \\
\text { economía circular, tomando en } \\
\text { consideración a los fabricantes, } \\
\text { minoristas, recicladores y gobierno que } \\
\text { deben seguir la legislación brasileña y } \\
\text { los acuerdos sectorial, con el fin de } \\
\text { revalorizar a los envases. }\end{array}$ \\
\hline
\end{tabular}

\begin{tabular}{llll}
\hline Sellitto & 3 industrias, Brasil & -Reciclar & Este estudio se destaca por la \\
(2018) & -Remanufactura & reutilización de residuos que se generan \\
& -Reutilizar & en la producción y post-consumo, la \\
& -Gestión de residuos & creación de centros populares de \\
& & reciclaje, reutilización de pallets y \\
& & menor utilización de material virgen. \\
& & Reduciendo el volumen de desechos en \\
& & vertederos y proporcionando una buena \\
& imagen corporativa (certificaciones)
\end{tabular}

\begin{tabular}{llll}
\hline $\begin{array}{l}\text { H\&M group } \\
\text { (2018) }\end{array}$ & $\begin{array}{l}\text { Sector textil, } \\
\text { Tiendas H\&M a } \\
\text { nivel mundial }\end{array}$ & $\begin{array}{l}\text {-Reciclar } \\
\text {-Remanufactura } \\
\text {-Reutilizar }\end{array}$ & $\begin{array}{l}\text { La compañía textil H\&M fabrica el } 57 \% \\
\text { de su producción total de materiales } \\
\text { reciclados otros materiales de origen } \\
\text { sostenible, disminuye las emisiones } \\
\text { CO2, aplica empaque circular, Afound } \\
\text { (nueva vida a productos no vendidos) y }\end{array}$ \\
& & $\begin{array}{l}\text { ofrece descuentos a los clientes por } \\
\text { devolver los envases vacíos o ropa no } \\
\text { deseada. }\end{array}$
\end{tabular}




\begin{tabular}{|c|c|c|c|}
\hline Autor/Año & Muestra/País & Variables & Resultados \\
\hline $\begin{array}{ll}\text { HP } & \text { Inc. } \\
(2017) & \end{array}$ & $\begin{array}{l}\text { Sector tecnológico, } \\
\text { Tiendas HP a nivel } \\
\text { mundial }\end{array}$ & $\begin{array}{l}\text {-Reciclaje } \\
\text {-Remanufactura } \\
\text {-Devolución } \\
\text {-Reutilización } \\
\text {-Gestión de residuos }\end{array}$ & $\begin{array}{l}\text { La logística inversa de HP captura el } \\
\text { valor de los materiales durante el mayor } \\
\text { tiempo posible, mediante la devolución, } \\
\text { reciclaje o reutilización de cartuchos } \\
\text { usados para producir otros nuevos. Se } \\
\text { caracteriza por aplicar deforestación } \\
\text { cero (solo material certificado y } \\
\text { reciclado). }\end{array}$ \\
\hline
\end{tabular}

Fuente: Elaboración propia.

En los trabajos analizados se evidenció que las grandes empresas están mejor preparadas para emplear la logística inversa y desplegar las actividades operativas requeridas. Así, las multinacionales HP del sector tecnológico y H\&M del sector textil, se identifican por su alto compromiso ambiental. Estas compañías realizan operaciones de reciclaje, remanufactura, reutilización, devolución y gestión de residuos. Se enfocan en reusar los desechos para convertirlos en nuevos productos. Por ejemplo, durante el 2018 H\&M group recogió 20.649 toneladas de textiles usados lo que es equivalente a 103 millones de nuevas camisetas. En contraposición, las pequeñas y medianas empresas tienen dificultades para gestionar prácticas organizacionales en el marco de la logística inversa, particularmente por la debilidad en reconocer sus beneficios para la sostenibilidad de las empresas.

Pagán et al. (2017), en su investigación conducida en supermercados, concluyen que la logística inversa conduce a una buena gestión empresarial, a través del efectivo manejo de residuos, reducción del uso de material virgen, menores costos para la producción y la preservación del medio ambiente, coincidiendo con Bottani et al. (2019) quienes indican que la esencia de la logística inversa es evitar que los desechos terminen en los vertederos, y como consecuencia se expanda la contaminación al planeta.

Se considera que la creciente concienciación de las personas y los gobiernos sobre la sostenibilidad social, económica y ambiental, ha obligado a las organizaciones a involucrase como actores principales en el cuidado ambiental mediante la aplicación de la logística inversa.

Las exigencias de los mercados dinámicos inciden mayormente a las organizaciones industriales, pues son las que habitualmente generan mayor contaminación. Las empresas exportadoras que participan en mercados con estrictas regulaciones del cuidado ambiental deben cambiar su flujo directo de productos por una logística inversa, lo cual favorece a la economía circular.

\section{Conclusión}

En consonancia con el objetivo planteado en esta investigación, concretamente, analizar la logística inversa como estrategia de diferenciación de las empresas exportadoras para competir en mercados dinámicos, mediante los resultados expuestos se han recogido las prácticas de logística inversa que aplican empresas que participan en los mercados internacionales, asociadas a diversos sectores y procedencia. 
Considerando que el dinamismo de los mercados y las crecientes preocupaciones ambientales marcan las nuevas tendencias comerciales, las empresas evidencian una creciente concienciación, lo que las ha llevado a involucrarse como actores principales en el cuidado del medio ambiental. Las empresas exportadoras, principalmente las concentradas en el sector industrial, como respuesta adaptada a las exigencias de los mercados que presentan estrictas regulaciones, están aplicando la logística inversa. Al mismo tiempo, dichas empresas se están involucrando en los esfuerzos por alcanzar los Objetivos del Desarrollo Sostenible planteado por la Unión Europea hasta el 2030. Siendo la Unión Europea un importante mercado, las empresas adoptan la logística inversa como estrategia para mejorar su imagen corporativa frente a los consumidores, $\mathrm{y}$, por tanto, diferenciarse de otros competidores.

La logística inversa aprovecha el valor de los productos fuera de uso, dándoles una nueva función o utilizándolos para la fabricación de nuevos productos. Hace posible la reducción de costos en la producción y empaquetado, disminuye el uso de materia prima virgen, mejora la relación entre distribuidor-cliente, establece una buena imagen de la empresa, y minimiza la huella ambiental, lo cual contribuye a la llamada economía circular.

En este sentido, la logística inversa tiene un amplio contexto de aplicación sobre las operaciones de reciclaje, devoluciones, gestión de residuos, remanufactura, análisis de costos, reutilización y redes de transporte. A partir de la aplicación de las prácticas que abarca la logística inversa, aumentan las posibilidades de diferenciación de la competencia. Dichas prácticas generan confianza y seguridad a los consumidores, específicamente con acciones enfocadas en las devoluciones de productos defectuosos, además asegura a la sociedad que sus desechos podrán ser reutilizados o eliminados de forma adecuada para que tenga el mínimo impacto ambiental. Actualmente, los consumidores están dispuestos a pagar más a condición de que lo que consumen tenga un manejo sostenible, aspecto que favorece a las empresas que aplican la logística inversa.

Señalando las limitaciones de este artículo que tiene un enfoque cualitativo, se considera en las futuras investigaciones realizar un estudio cuantitativo de empresas exportadoras que apliquen la logística inversa como una estrategia de diferenciación hacia mercados exigentes. Una investigación adicional sería el estudio de la logística inversa como una estrategia competitiva de liderazgo en costos.

\section{Bibliografía}

Agrawal, S., \& Singh, R. (2019). Analyzing disposition decisions for sustainable reverse logistics: Triple Bottom Line approach. Resources, Conservation \& Recycling, 150, 2-11.

Alshubiri, F. (2017). The Impact of Green Logistics-Based Activities on the Sustainable Monetary Expansion Indicators of Oman. Journal of Industrial Engineering and Management, 10(2), 388-405.

Anca, V. (2019). Logistics and supply chain management: An overview. Studies in Business and Economics, 14(2), 209-215. 
Antonyová, A., Antony, P., \& Soewito, B. (2016). Logistics Management: New trends in the Reverse Logistics. Journal of Physics: Conference Series, 710(1), 1-10.

Ballou, R. (2004). Logística Administración de la cadena de suministro. México: Pearson aducation.

Bastos, S. K., \& Gallego, A. (2016). ¿Qué está cambiando el rumbo de la logística? Cinco tendencias logísticas en la cadena de suministro. Mercatec(51), 19-27.

Bottani, E., Vignali, G., Mosna, D., \& Montanari, R. (2019). Economic and environmental assessment of different reverse logistics scenarios for food waste recovery. Sustainable Production and Consumption, 20, 289-303.

Brand, S. E., Monsalve, F. L., \& Rojas, D. E. (2017). Caracterización de Logística en las empresas del Altiplano del Oriente Antioqueño. Revista Loginn, 1(1), $29-42$.

Brüning, S. L., Masi, D., Jacobsen, P., \& Godsell, J. (2018). How the reverse supply chain contributes to a firm's competitive strategy: a strategic alignment perspective. Production Planning \& Control, 29(6), 452-463.

Bustos F., C. (2015). La logística inversa como fuente de producción sostenible. Actualidad Contable FACES, 18(30), 7-32.

Camargo, R. S., Franco, L. J., Chud, P. V., \& Osorio, G. J. (2017). Modelo de simulación dinámica para evaluar el impacto ambiental de la producción y logística inversa de las llantas. Ingeniería y Desarrollo, 35(2), 357-381.

Carrillo, H. K. (2017). Estrategias Sustentables en Logística y Cadenas de Suministro. Revista Loginn, 1(1), 53-61.

Carro, P. R., \& Gónzalez, G. D. (2013). Logística empresarial. Mar de la plata: Apuntes de estudio.

Castellanos, R. A. (2015). Logística comercial internacional. Barranquilla, Colombia: Ecoe Ediciones.

Dias Marques, M., Pagán, M. M., Silva, B. J., Cataneo, P. F., \& Da Silva, D. (2017). Logística inversa de envases de plaguicidas: percepción de los pequeños y medianos productores agrícolas. Exacta, 15(2), 353-368.

DW. (28 de Noviembre de 2019). Obtenido de https:/www.dw.com/es/uni\%C3\%B3n-europealos-detalles-de-la-proclamaci\%C3\%B3n-de-la-emergencia-clim\%C3\%A1tica/a51456909

Flamarique, S. (2017). Gestión de operaciones de almacenaje. Marge Books. Obtenido de http://ebookcentral.proquest.com/lib/utmachalasp/detail.action?docID=5045328.

González, G. P., \& Gutiérrez, G. M. (2018). Logistica de aprovisionamiento. Madrid: SÍNTESIS.

Guarnieri, P., Cerqueira-Streit, J., \& Batista, L. (2020). Reverse logistics and the sectoral agreement of packaging industry in Brazil towards a transition to circular economy. Resources, Conservation \& Recycling, 153, 2-12.

Esta obra se comparte bajo la licencia Creative Common Atribución-No Comercial 4.0 International (CC BY-NC 4.0) 
Gullifa, S., Jatib, M., Marcuzzi , A., \& Pérez, C. (2017). Optimización de la logística inversa en el reciclado de envases. Reddi, 2(2), 1-12.

H\&M group. (2018). H\&M. Obtenido de https://about.hm.com/content/dam/hmgroup/groupsite/documents/masterlanguage/CSR/re ports/2018_Sustainability_report/HM_Group_SustainabilityReport_2018_\%20FullReport .pdf

HP Inc. (2017). HP home. Obtenido de https://www8.hp.com/h20195/v2/GetPDF.aspx/c05364027.pdf

Huérfano, P. E., \& Meleán, R. R. (2017). Logística inversa: Estrategias de recuperación en empresas zulianas de derivados lácteos. InnovaG, 3, 37-44.

Jeszka , A. (2014). Returns management in the supply chain. Scientific Journal of Logistics, 10(3), 295-304.

Job, M., \& Nyongesa, M. (2016). Product differentiation strategy for competitive advantage in kenya cooperative creameries. Africa International Journal of Management Education and Governance (AIJMEG), 1(3), 1-9.

Lechner, G., \& Reimann, M. (2019). Integrated decision-making in reverse logistics: an optimisation of interacting acquisition, grading and disposition processes. International Journal of Production Research, 57, 2-20.

Liu, W., Wang, D., Long, S., \& Shen, X. (2019). Service supply chain management: a behavioural operations perspective. Modern Supply Chain Research and Applications, $1(1), 28-53$.

Lopes, D. M., D'Agosto, M., Ferreira, A., \& Oliveira, C. (2014). ) Improving post-sale reverse logistics in department stores: a Brazilian case study. Journal of Transport Literature, $8(2), 325-348$.

Magee, J. (1967). Industrial logistics. McGraw-Hill.

Maquera, G. (2012). Logística verde e Inversa, Responsabilidad Universitaria Socioambiental, Corporativa y Productividad. Revista Apuntes Universitarios, 2(1), 31-54.

Martínez, V. J., De la hoz, B. J., García, G. J., \& Molina, I. (2017). Gestión logística en Pymes del sector de operadores de carga del Departamento del Atlántico. Revista Espacios, 38(58), 1-6.

Mejía, M. J., San Andrés, R. P., \& Paredes, C. I. (2018). Logística y canales de distribución para la mejora de procesos. Estudio de Caso NEDERAGRO SA- Período 2019. Innova, 3(6), $155-167$.

Mendoza, R. C., Alfaro, D. J., \& Paternina, A. C. (2016). Manual práctico para gestión logística: envase y embalaje, transporte y cadena de frío, preservación de productos del agro. Barranquilla: Universidad del Norte.

Michlowicz, E., \& Smolińska, K. (2015). Research on the flow of material in production logistics. Research in Logistics \& Production, 5(1), 21-31. 
Morgan, T., Tokman, M., Richey, R. G., \& Defee, C. (2018). Resource commitment and sustainability: a reverse logistics performance process model. International Journal of Physical Distribution \& Logistics Management, 48(2), 164-182.

Noé, A. C. (2015). Relación entre logística inversa y desempeño. Cuadernos de Administración, $31(53), 85-96$.

Nurul, I. H., Zwolinski, P., Mandil, G., \& Brissaud, D. (2017). Decision making system for designing products and production systems for remanufacturing activities. Procedia CIRP, 61, $212-217$.

Pagán, M. M., Tonelli, S. D., Silva, B. J., \& Da Silva, D. (2017). La logística inversa como herramienta para la gestión de residuos de los supermercados de venta al por menor. Revista de Gestão Ambiental e Sustentabilidade, 6(3), 150-165.

Palau, C. J. (2015). Nuevas tendencias en logística. Business school, 4-45.

Peña, A. C. (2016). Manual de transporte para el comercio internacional: selección y gestión del transporte para la exportación. Barcelona: Marge Books. Obtenido de http://ebookcentral.proquest.com/lib/utmachalasp/detail.action?docID=5045316.

Philomena, M. H. (2018). A study of evolution and future of supply chain management. IRJMST: International Research Journal of Management Sciences \& Technology, 9(11), 13-17.

Pinheiro de Lima, O., Breval, S. S., Rodríguez, T. C., \& Follmann, N. (2016). Una nueva definición de la logística interna y forma de evaluar la misma. Ingeniare. Revista chilena de ingeniería, 25(2), 264-276.

Rubio, S., \& Jiménez-Parra, B. (2014). Reverse Logistics: Overview and Challenges for Supply Chain Management. International Journal of Engineering Business Management, 6(12), $1-7$.

Rubio, S., Jiménez-Parra, B., Chamorro-Mera, A., \& Miranda, F. (2019). Reverse Logistics and Urban Logistics: Making a Link. Sustainability, 11(20), 2-17.

Santana, M. R. (2018). La logística inversa y su importancia para la organización y sostenibilidad del medio ambiente. Revista científica multidisciplinaria base de conocimiento, 4, 36-51.

Saravanan, S., Sathiyagothai, B., \& Manigandan, K. (2017). Future Benefits of Reverse Logistics: A Study for Long-Run of A Company. IJRASET, 5(6), 1242-1247.

Sellitto, M. A. (2018). Reverse logistics activities in three companies of the process industry. Journal of Cleaner Production, 187, 923-931.

Semuel, H., Siagian, H., \& Octavia, S. (2017). The effect of leadership and innovation ondifferentiation strategy and company performance. Procedia - Social and Behavioral Sciences, 237, $1152-1159$.

Servera-Francés, D. (2010). Concepto y evolución de la función logística. INNOVAR. Revista de Ciencias Administrativas y Sociales, 20(38), 217-234. 
Singh, J., Shani, A., Femal, H., \& Deif, A. (2016). Packaging's Role in Sustainability: Reusable Plastic Containers in the Agricultural-Food Supply Chains. Organizing Supply Chain Processes for Sustainable Innovation in the Agri-Food Industry, 5, 175-204.

Sorlózano, G. M. (2018). Optimización de la cadena logística. Malaga: IC Editorial.

Speranza, M. (August de 2016). Trends in transportation and logistics. European Journal of Operational Research, 264(3), 830-836.

Trochu, J., Chaabane, A., \& Ouhimmou, M. (2019). A carbon-constrained stochastic model for eco-efficient reverse logistics network design under environmental regulations in the CRD industry. Journal of Cleaner Production, 1-51.

Vega de la Cruz, L., Marrero, F. C., \& Pérez, P. M. (2017). Contribución a la logística inversa mediante la implantación de la reutilización por medio de las redes de Petri. Revista chilena de ingeniería, 25(1), 154-169.

Yousefloo, A., \& Babazadeh, R. (2019). Designing an integrated municipal solid waste management network: A case study. Journal of Cleaner Production, 1-36. 